\title{
Some Equal Degree Graph Edge Chromatic Number
}

\author{
Jun Liu ${ }^{1, a}$, Zhi Guo Ren ${ }^{1}$, Qiu Ju Yue ${ }^{1}$, Zhong Yi Feng ${ }^{1}$ and Cai Hui Lan ${ }^{1}$ \\ 1. Lanzhou City University, School of Information Science and engineering, Lanzhou 730070, P.R. China
}

\begin{abstract}
Let $G(V, E)$ be a simple graph and $k$ is a positive integer, if it exists a mapping of $f$, and satisfied with $f\left(e_{1}\right) \neq 6=f\left(e_{2}\right)$ for two incident edges $e_{1}, e_{2} \in E(G), f\left(e_{1}\right) \neq 6=f\left(e_{2}\right)$, then $f$ is called the $k$-proper-edge coloring of $G\left(k-P E C\right.$ for short).The minimal number of colors required for a proper edge coloring of $G$ is denoted by $X^{\prime}(G)$ and is called the proper edge chromatic number. It exists a $k$-proper edge coloring of simple graph of $G$, for any two adjacent vertices $u$ and $v$ in $G$, the set of colors assigned to the edges incident to $u$ differs from the set of colors in cident to $v$, then $f$ is called $k$-adjacent-vertex-distinguishing proper edge coloring, is avvreviated $k$-AVDEC, also called a adjacent strong edge coloring. The minimal number of colors required for a adjacent-vertex-distinguishing edge coloring of $G$ is denoted by $X_{a d}^{\prime}(G)$ and is called adjacent-vertex-distinguishing edge chromatic number. The new class graphs of equal degree graph are be introduced, and this class graphs adjacent-vertex-distinguishing edge chromatic numbers of path ,cycle, fan, complete graph, wheel, star are presented in this paper.
\end{abstract}

\section{Introduction}

The coloring problem of graphs is a extremely difficult problem, widely applied in practice. In [1], some conditional coloring problems are introduced. Some network problems can be converted to the edge coloring $^{[2-9]}$ and adjacent strong edge coloring.

Definition $1^{[10]}$ For a $\operatorname{graph} G(V, E)$, if a proper coloring $f$ is satisfied with $C(u) \neq C(v)$ for $u, v \in V(G)(u \neq v)$ and $u v \in E(G)$ then $f$ is called $\mathrm{k}$-adjacent-vertex- distinguishing edge coloring of $G$, is abbreviated $k-A V D E C$, and

$$
X_{a d}^{\prime}(G)=\min \{k \mid k-A V D E C o f G\}
$$

is called the adjacent-vertex-distinguishing edge chromatic number of $G^{[6]}$, where $C(u)=\{f(u v) \mid u v \in E(G)$

Conjecture ${ }^{[2]}$ Let $G$ be a connected graph with $|G| \geq 3$, and $G \neq C_{5}(5-$ cycle $)$, then

$$
X_{a d}^{\prime}(G) \leq \Delta(G)+2
$$

where $\Delta(G)$ is the maximum degree of graph $G$.

Definition $2^{[5]} \operatorname{Let} G(V, E)$ be a simple graph, $M(G)$ is called the equal degree graph of $G$, where

$$
V(M(G))=V(G) \bigcup V^{\prime} \bigcup\{W\} ;
$$

$$
\begin{aligned}
& E(M(G))=E(G) \bigcup\left\{u v^{\prime} \mid u \in V(G),\right. \\
& \left.v^{\prime} \in V^{\prime}, d(u)=d(v)\right\} \bigcup\left\{w v^{\prime} \mid v^{\prime} \in V^{\prime}\right\}
\end{aligned}
$$

Where as

$$
V^{\prime}=\left\{v^{\prime} \mid v \in V(G)\right\},\{w\} \bigcap\left(V(G) \quad \vee V^{\prime}\right)=\varphi .
$$

\section{Main result}

Lemma 1For $n \geq 2$, then

Lemma 2 For $n \geq 3$, then

$$
\Delta\left(M\left(P_{n}\right)\right)=\left\{\begin{array}{l}
3, n=2,3 ; \\
n, n \geq 4 .
\end{array}\right.
$$

$$
\Delta\left(M\left(P_{n}\right)\right)=n+2
$$

Lemma 3 For $n \geq 3$, then

$$
\Delta\left(M\left(F_{n}\right)\right)=\left\{\begin{array}{l}
5, n=2,3 \\
n+1, n \geq 4 .
\end{array}\right.
$$

Lemma 4 For $n \geq 2$, then

$$
\Delta\left(M\left(K_{n}\right)\right)=\left\{\begin{array}{l}
3, n=2 \\
5, \quad N=3 \\
2 n-1 \geq 4 .
\end{array}\right.
$$

Lemma 5 For $n \geq 3$, then

$$
\Delta\left(M\left(W_{n}\right)\right)=\left\{\begin{array}{l}
7, n=3 \\
n+3, n \geq 4 .
\end{array}\right.
$$

authore-mail: 527876625@.qq.com

This studywas supported by Lanzhou City University Ph.D. Research Fund (21265099,41361013,GS[2013]GHB1084, LZCU-BS2013-09 and LZCU-BS2013-12). 
Lemma 6 For $n \geq 3$, then

$$
\Delta\left(M\left(S_{n}\right)\right)=\left\{\begin{array}{l}
3, n=1,2 \\
n+1, n \geq 3 .
\end{array}\right.
$$

Lemma ${ }^{[2]}$ Let $G$ be a connected simple graph with $|V(G)| \geq 3$, if $u v \in E(G)$ and $d(u)=d(v)=\Delta(G)$, then

$$
X_{a d}^{\prime}(G) \geq \Delta(G)+1
$$

Theorem 1 For $n \geq 2$, then

$$
X_{a d}^{\prime}\left(M\left(P_{n}\right)\right)=\left\{\begin{array}{l}
5, n=3 ; \\
n+1, n \geq 4 .
\end{array}\right.
$$

Proof Let

$V\left(P_{n}\right)=\left\{u_{1}, u_{2}, \cdots, u_{n}\right\}, V^{\prime}=\left\{v_{1} v_{2}, \cdots, v_{n}\right\}, \quad$ there are two cases to be discussed as follow:

Case1When $n=2, \Delta\left(M\left(P_{2}\right)\right)=3$ from lemma 1 . However $u_{1}, u_{2}, v_{1}, v_{2}$ are vertices which are maximum degree of $M\left(P_{2}\right)$ and all adjacent, $X_{a d}^{\prime}\left(M\left(P_{2}\right)\right) \geq$ $\Delta\left(M\left(P_{n}\right)\right)=4$ from lemma 7. If $M\left(P_{2}\right)$ exists $4-A V D E C$, because four vertices of maximum degree are adjacent each other, and $\left(\begin{array}{l}4 \\ 3\end{array}\right)=4$, the set are $\{1,2,3\},\{1,2,4\}$, $\{1,3,4\},\{2,3,4\}$. Let every set correspond a vertex, we can obtain the vertices of coloring same color are odd, but the vertices of coloring same color are even by graph theory, so $M\left(P_{2}\right)$ don't exists 4-AVDEC. In order to prove the conclusion be true, we only need construct a map $f$ from $E\left(M\left(P_{2}\right)\right)$ to $\{1,2,3,4,5\}$ :

$$
\begin{aligned}
& f\left(u_{1} v_{1}\right)=2 ; \\
& f\left(u_{2} v_{2}\right)=3 ; \\
& f\left(u_{1} u_{2}\right)=4 ; \\
& f\left(w v_{1}\right)=3 ; \\
& f\left(w v_{2}\right)=5 \\
& \text { Then we have } \\
& \bar{C}\left(u_{1}\right)=\{3,5\} ; \\
& \bar{C}\left(u_{2}\right)=\{2,5\} ; \\
& \bar{C}\left(v_{1}\right)=\{4,5\} ; \\
& \bar{C}\left(v_{2}\right)=\{2,4\} ;
\end{aligned}
$$$$
f\left(u_{1} v_{2}\right)=f\left(u_{2} v_{1}\right)=1 \text {; }
$$

Obviously, the $f$ is 5 -AVDEC of $M\left(P_{2}\right)$, the conclusion is true.

When $n=3, \Delta\left(M\left(P_{3}\right)\right)=3$ from lemma 1. But $M\left(P_{2}\right) \subset M\left(P_{3}\right), M\left(P_{3}\right) \geq 5$ from $\mathrm{n}=2$. It is obviously to prove exists $5-A V D E C$ of $M\left(P_{3}\right)$, we only need to construct a map $f$ from $E\left(M\left(P_{3}\right)\right)$ to $\{1,2,3,4,5\}$ :

$$
\begin{aligned}
& f\left(u_{1} u_{2}\right)=f\left(u_{3} v_{3}\right)=1 ; \\
& f\left(u_{1} v_{1}\right)=f\left(u_{2} u_{3}\right)=2 ; \\
& f\left(u_{1} v_{3}\right)=f\left(w v_{2}\right)=3 ; \\
& f\left(u_{1} u v_{2}\right)=f\left(w v_{1}\right)=4 ; \\
& f\left(u_{3} v_{1}\right)=f\left(w v_{3}\right)=5 ;
\end{aligned}
$$

Then we have

$\bar{C}\left(u_{1}\right)=\{4,5\}$;

$\bar{C}\left(u_{2}\right)=\{3,5\}$;

$\bar{C}\left(u_{3}\right)=\{3,4\}$;

$$
\begin{aligned}
& \bar{C}\left(v_{1}\right)=\{1,3\} ; \\
& \bar{C}\left(v_{2}\right)=\{1,2,5\} ; \\
& \bar{C}\left(v_{3}\right)=\{2,4\} ;
\end{aligned}
$$

Obviously, the $f$ is 5 -AVDEC of $M\left(P_{3}\right)$, the conclusion is true.

Case2 When $n \geq 4, \Delta\left(M\left(P_{n}\right)\right)=n$ from lemma 1 . However the vertices of $u_{2}, u_{3}, \ldots, u_{n-1}, \mathrm{w}$ are vertices which are maximum degree, and exist some are adjacent, $X_{a d}^{\prime}\left(M\left(P_{n}\right)\right) \geq n+1$ from lemma 7 . It is obviously to prove exists $(n+1)-A V D E C$ of $M\left(P_{n}\right)$, we only need to construct a map $f$ from $E\left(M\left(P_{n}\right)\right)$ to $\{1,2, \cdots$, $n, 0\}$ :

$$
\begin{aligned}
& f\left(u_{i} u_{i+1}\right)=i-1, i=1,2, \cdots, n-1 ; \\
& f\left(u_{1} v_{1}\right)=1 ; f\left(u_{1} v_{n}\right)=2 ; \\
& f\left(u_{i} v_{j}\right)=i+j-2(\bmod n), \\
& i=2,3, n-1 ; j=2,3, n-1 ; \\
& f\left(u_{n} v_{1}\right)=n ; f\left(u_{n} v_{n}\right)=0 ; \\
& f\left(w v_{i}\right)=i-1, i=1,2, \cdots, n .
\end{aligned}
$$

So we have

$$
\begin{aligned}
& \bar{C}\left(v_{i}\right)=\{0,1,2\} ; \\
& \bar{C}\left(u_{2}\right)=\{n\} ; \\
& \bar{C}\left(u_{i}\right)=\{i-3\}, i=3,4, \cdots, n-1 ; \\
& \bar{C}\left(u_{n}\right)=\{0, n-2, n-1\} ; \\
& \bar{C}\left(v_{1}\right)=\{0,1, n\} ; \\
& \bar{C}\left(v_{i}\right)=\{i-1, i, \cdots, n+i-3\}(\bmod n+1), \\
& i=2,3, \cdots, n-1 ; \\
& \bar{C}\left(v_{n}\right)=\{0,2, n-1\} .
\end{aligned}
$$

Obviously, the $f$ is $(n+1)-A V D E C$ of $M\left(P_{n}\right)$, the conclusion is true.

From all of above two cases, the conclusion is true.

Theorem 2 For $n \geq 3$, then

$$
X^{\prime}\left(M\left(C_{n}\right)\right)=n+3 \text {. }
$$

Proof Let

$V\left(C_{n}\right)=\left\{u_{1}, u_{2}, \cdots, u_{n}\right\}, V^{\prime}=\left\{v_{1}, v_{2}, \cdots, v_{n}\right\}, u_{1}, u_{2}, \cdots, u_{n}$ are vertices of maximum degree andexists some adjacent, $X_{a d}^{\prime}\left(M\left(C_{n}\right)\right) \geq n+3$ from lemma 2 and 7. It is obviously to prove exists $(n+3)-A V D E C$ of $\geq M\left(C_{n}\right)$, we only need to construct a map $f$ from $E\left(M\left(C_{n}\right)\right)$ to $\{1,2, \cdots, n+2,0\}$ :

$$
\begin{aligned}
& f\left(u_{i} u_{i+1}\right)=i-1, i=1,2, \cdots, n-1 ; \\
& f\left(u_{1} u_{n}\right)=0 ; \\
& f\left(u_{i} v_{j}\right)=i+j(\bmod n+3), \\
& i=1,2, \cdots, n-1 ; j=1,2, \cdots n ; \\
& f\left(u_{n} v_{j}\right)=n+j, j=1,2 ; \\
& f\left(u_{n} v_{j}\right)=j-2, j=3,4, \cdots, n ; \\
& f\left(w v_{j}\right)=j-1 .
\end{aligned}
$$

So we have

$\bar{C}\left(u_{1}\right)=\{n+2\}$;

$\bar{C}\left(u_{i}\right)=\{i-1\}, i=2,3, \cdots, n-1$;

$\bar{C}\left(u_{n}\right)=\{n\}$. 
Obviously, the $f$ is $(n+3)-A V D E C$ of $M\left(C_{\mathrm{n}}\right)$, the conclusion is true.

Theorem 3 For $n \geq 3$, then

$$
X^{\prime}\left(M\left(F_{n}\right)\right)=\left\{\begin{array}{l}
6, n=2,3 \\
n+2, n \geq 4
\end{array}\right.
$$

Proof Let

$V\left(F_{n}\right)=\left\{u_{0}, u_{1}, u_{2}, \cdots, u_{n}\right\}, V^{\prime}=\left\{v_{0}, v_{1}, v_{2}, \cdots, u_{n}\right\}$, there are three cases to be discussed as follow:

Case1 When $n=2, M\left(F_{2}\right)=M\left(C_{3}\right)$, so we can obtain $X^{\prime}\left(M\left(F_{3}\right)\right)=X^{\prime}\left(M\left(C_{3}\right)\right)=6$, then the conclusion is true.

Case2 When $n=3, \Delta\left(M\left(F_{3}\right)\right)=5$ from lemma 3 , and $M\left(F_{3}\right)$ exists two vertices of maximum degree are adjacent, so $X_{a d}^{\prime}\left(M\left(F_{3}\right)\right) \geq 6$ by lemma 7 . It is obviously to prove exists $6-A V D E C$ of $M\left(F_{3}\right)$, we only need to construct a map $f$ from $E\left(M\left(F_{3}\right)\right)$ to $\{1,2, \cdots, 5,0\}$ :

$$
\begin{aligned}
& f\left(u_{0} u_{i}\right)=i-1, i=1,2,3 \\
& f\left(u_{i} u_{i+1}\right)=i+1, i=1,2 ; \\
& f\left(u_{0} v_{0}\right)=3 ; \\
& f\left(u_{0} v_{2}\right)=4 ; \\
& f\left(u_{1} v_{1}\right)=3 \\
& f\left(u_{3} v_{1}\right)=4 ; \\
& f\left(u_{3} v_{3}\right)=5 ; \\
& f\left(w v_{i}\right)=i-1, i=0,1,2,3 .
\end{aligned}
$$

The we have

$\bar{C}\left(u_{0}\right)=\{5\}$;

$\bar{C}\left(u_{2}\right)=\{0\}$. is true.

Obviously, the $f$ is $6-A V D E C$ of $M\left(F_{3}\right)$, the conclusion

Case 3 When $n \geq 4, \Delta\left(M\left(F_{n}\right)\right)=n+1$ from lemma $3, M\left(F_{\mathrm{n}}\right)$ exists some vertices of maximum degree are adjacent, $X^{\prime}\left(M\left(F_{\mathrm{n}}\right)\right) \geq \Delta\left(M\left(F_{n}\right)\right)+1=n+2$ by lemma 3,7 . It is obviously to prove exists $(n+2)-A V D E C$ of $M\left(F_{n}\right)$, we only need to construct a map $f$ from $E\left(M\left(F_{n}\right)\right)$ to $\{1,2, \cdots$, $n+1,0\}$ :

$$
\begin{aligned}
& f\left(u_{0} u_{i}\right)=i-1, i=1,2, \cdots, n ; \\
& f\left(u_{i} u_{i+1}\right)=i+1, i=1,2 \cdots, n-1 ; \\
& f\left(u_{0} v_{0}\right)=n ; \\
& f\left(u_{1} v_{1}\right)=3 ; \\
& f\left(u_{1} v_{n}\right)=4 ; \\
& f\left(u_{i} v_{j}\right)=i+j(\bmod n+2), \\
& i=2,3, \cdots, n-1 ; j=2,3, \cdots, n-1 ; \\
& f\left(u_{n} v_{1}\right)=1 ; \\
& f\left(u_{n} v_{n}\right)=2 ; \\
& f\left(w v_{i}\right)=i+1, i=0,1, \cdots, n . \\
& \text { So we have } \\
& \bar{C}\left(u_{0}\right)=\{n+2\} ; \\
& \bar{C}\left(u_{i}\right)=\{i-2\}, i=2,3, \cdots, n-1 ; \\
& \bar{C}(w)=\{0\} . \\
& \text { Obviously, the } f \text { is }(n+2)-A V D E C \text { of } M\left(F_{n}\right), \text { the } \\
& \text { conclusion is true. } \\
& \text { From all of above, the conclusion is true. }
\end{aligned}
$$

Theorem 4 For $n \geq 2$, then

$$
X^{\prime}\left(M\left(K_{n}\right)\right)=\left\{\begin{array}{l}
5, n=2 \\
6, n=3 \\
\Delta\left(M\left(K_{n}\right)\right)+1, n \geq 4 .
\end{array}\right.
$$

Proof Let

$V\left(F_{n}\right)=\left\{u_{1}, u_{2}, u_{2}, \cdots, u_{n}\right\}, V^{\prime}=\left\{v_{1}, v_{2}, \cdots, u_{n}\right\}$, there are three cases to be discussed as follow:

Case 1 When $n=2, M\left(K_{2}\right)=M\left(P_{2}\right)$, so we can obtain $X^{\prime}\left(M\left(K_{2}\right)\right)=X^{\prime}\left(M\left(P_{2}\right)\right)=5$, the conclusion is true.

Case 2 When $n=3, M\left(K_{3}\right)=M\left(C_{3}\right)$, so we can obtain $X^{\prime}\left(M\left(K_{3}\right)\right)=X^{\prime}\left(M\left(C_{3}\right)\right)=6$, the conclusion is true.

Case 3 When $n \geq 4, \Delta\left(M\left(K_{n}\right)\right)=2 n-1$ from lemma $4, M\left(K_{n}\right)$ exists some vertices of maximum degree are adjacent, $X^{\prime}\left(M\left(K_{n}\right)\right) \geq \Delta\left(M\left(K_{n}\right)\right)+1=2 n$ by lemma 4,7 . It is obviously to prove exists $2 n-A V D E C$ of $M\left(K_{n}\right)$,we only need to construct a map $f$ from $E\left(M\left(K_{n}\right)\right)$ to $\{1,2, \cdots, 2 n-1,0\}$ :

$$
\begin{aligned}
& f\left(u_{i} u_{j}\right)=i+j, \\
& i=1,2, \cdots, n-1 ; j=i+1, i+2 \cdots, n-1 ; \\
& f\left(u_{i} v_{j}\right)=i+j+n-3(\bmod 2 n), \\
& i=1,2, \cdots, n ; j=1,2, \cdots, n-1 ; \\
& f\left(v_{n} u_{1}\right)=2 n-1 ; \\
& f\left(v_{n} u_{i}\right)=2(i-1)-1, i=2,3, \cdots, n ; \\
& f\left(w v_{n}\right)=i, i=1,2, \cdots, n-1 ; \\
& f\left(w v_{n}\right)=n, n \equiv 0(\bmod 2) ; \\
& f\left(w v_{n}\right)=n+1, n \equiv 1(\bmod 2) .
\end{aligned}
$$

The we have

$$
\bar{C}\left(u_{i}\right)=\{2 n+i-3\}(\bmod 2 n), i=1,2, \cdots, n .
$$

Obviously, the $f$ is $2 n-A V D E C$ of $M\left(K_{n}\right)$, the conclusion is true.

From all of above, the conclusion is true.

Theorem 5 For $n \geq 3$, then

$$
X^{\prime}\left(M\left(W_{n}\right)\right)=\left\{\begin{array}{l}
8, n=3 \\
n+4, n \geq 4 .
\end{array}\right.
$$

Proof Let

$V\left(W_{n}\right)=\left\{u_{0}, u_{1}, u_{2}, \cdots, u_{n}\right\}, V^{\prime}=\left\{v_{0}, v_{1}, v_{2} \cdots, u_{n}\right\}$, there are two cases to be discussed as follow:

Case1 When $n=3, M\left(W_{3}\right)=M\left(K_{4}\right)$, so we can obtain $X^{\prime}\left(M\left(W_{3}\right)\right)=X^{\prime}\left(M\left(K_{4}\right)\right)=8$, the conclusion is true.

Case2 When $n \geq 4, \Delta\left(M\left(W_{n}\right)\right)=n+3$ from the lemma $5, M\left(W_{n}\right)$ exists some vertices of maximum degree

are adjacent, $X^{\prime}\left(M\left(W_{n}\right)\right) \geq \Delta\left(M\left(W_{n}\right)\right)+1=n+4$ by lemma 5,7 . It is obviously to prove exists $(n+4)-A V D E C$ of $M\left(W_{n}\right)$, we only need to construct a map $f$ from $E\left(M\left(W_{n}\right)\right)$ to $\{1,2, \cdots, n+3,0\}$ :

$$
\begin{aligned}
& f\left(u_{0} u_{i}\right)=i-1, i=1,2, \cdots, n ; \\
& f\left(u_{0} v_{0}\right)=n ; \\
& f\left(u_{i} v_{j}\right)=i+j-1(\bmod n+4), \\
& i=1,2, \cdots, n-1 ; j=1,2, \cdots n ; \\
& f\left(u_{n} v_{1}\right)=n ; \\
& f\left(u_{n} v_{j}\right)=n+j(\bmod n+4), j=2,3, \cdots, n-1 ; \\
& f\left(u_{n} v_{n}\right)=n-3 ;
\end{aligned}
$$




$$
\begin{aligned}
& f\left(u_{i} u_{i+1}\right)=n+i+1(\bmod n+4), \\
& i=1,2, \cdots, n-1 ; \\
& f\left(u_{1} u_{n}\right)=n+1 ; \\
& f\left(w v_{i}\right)=n+2+j(\bmod n+4), i=0,1, \cdots n, .
\end{aligned}
$$

Then we have

$\bar{C}\left(u_{i}\right)=\{n+i+2\}(\bmod n+4), i=1,2, \cdots, n$.

Obviously, the $f$ is $(n+3)-A V D E C$ of $M\left(W_{n}\right)$, the conclusion is true.

From all of above, the conclusion is true.

Theorem 6 For $n \geq 1$, then

$$
X^{\prime}\left(M\left(S_{n}\right)\right)=\left\{\begin{array}{l}
5, n=1,2 \\
n+3, n \geq 3 .
\end{array}\right.
$$

Proof Let

$V\left(S_{n}\right)=\left\{u_{0}, u_{1}, u_{2}, \cdots, u_{n}\right\}, V^{\prime}=\left\{v_{0}, v_{1}, v_{2} \cdots, u_{n}\right\}$, there are three cases to be discussed as follow:

Case 1 When $n=1, M\left(S_{1}\right)=\mathrm{M}\left(P_{2}\right)$, so we can obtain $X^{\prime}\left(M\left(S_{1}\right)\right)=X^{\prime}\left(M\left(P_{2}\right)\right)=5$, the conclusion is true.

Case 2 When $n=2, M\left(S_{2}\right)=M\left(P_{3}\right)$, so we can obtain $X^{\prime}\left(M\left(S_{2}\right)\right)=X^{\prime}\left(M\left(P_{3}\right)\right)=6$, the conclusion is true.

Case 3 When $n \geq 3, \Delta\left(M\left(S_{n}\right)\right)=n+1$ by lemma $6, M\left(S_{n}\right)$ exists some vertices of maximum degree are adjacent, $X^{\prime}\left(M\left(S_{n}\right)\right) \geq \Delta\left(M\left(S_{n}\right)\right)+1=\mathrm{n}+2$ by lemma 6,7 . If $M\left(S_{n}\right)$ exists $n+2-A V D E C$, then

$$
\begin{aligned}
& \bar{C}\left(u_{0}\right)=\bar{C}\left(u_{i}\right)=\bar{C}\left(v_{i}\right)=1, i=1,2, \cdots n . \\
& \left\{\begin{array}{l}
\bar{C}\left(u_{0}\right) \in \bar{C}\left(u_{1}\right) \cup \bar{C}\left(u_{2}\right) \cup \cdots \cup \bar{C}\left(u_{n}\right) ; \\
\bar{C}\left(u_{1}\right) \in \bar{C}\left(v_{1}\right) \cup \bar{C}\left(u_{2}\right) \cup \cdots \cup \bar{C}\left(v_{n}\right) \\
\bar{C}\left(u_{2}\right) \in \bar{C}\left(v_{1}\right) \cup \bar{C}\left(v_{2}\right) \cup \cdots \cup \bar{C}\left(v_{n}\right) \\
\cdots \\
\bar{C}\left(u_{n}\right) \in \bar{C}\left(v_{1}\right) \cup \bar{C}\left(v_{2}\right) \cup \cdots \cup \bar{C}\left(v_{n}\right)
\end{array}\right.
\end{aligned}
$$

from (1) and (2), we can obtain

$$
\mid \bar{C}\left(u_{0}\right)+\bar{C}\left(u_{1}\right)+\bar{C}\left(u_{2}\right)+\ldots+\bar{C}\left(u_{n}\right)+\bar{C}\left(v_{1}\right)+\bar{C}\left(v_{2}\right)+\ldots
$$

$+\bar{C}\left(v_{n}\right) \mid>n+2$.

It is obvious that $M\left(S_{n}\right)$ don't exists $n+2-A V D E C$. In order to prove the conclusion be true, we only need to construct a map $f$ from $E\left(M\left(S_{n}\right)\right)$ to $\{1,2, \cdot$. , $n+2,0\}$ :

$$
\begin{aligned}
& f\left(u_{0} u_{i}\right)=i-1, i=1,2, \cdots, n ; \\
& f\left(u_{0} v_{0}\right)=n ; \\
& f\left(u_{i} v_{j}\right)=i+j(\bmod n+3), \\
& i=1,2, \cdots, n ; j=1,2, \cdots n ; \\
& f\left(w v_{i}\right)=i-1, i=0,1, \cdots, n
\end{aligned}
$$

Then we have

$\bar{C}\left(u_{0}\right)=\{n+1, n+2\}$;

$\bar{C}\left(u_{i}\right)=\{1, n+2\}$;

$\bar{C}\left(u_{i}\right)=\{i-2, i\}, i=2,3, \cdots, n$;
$\bar{C}\left(v_{1}\right)=\{0, n+2\} ;$

$\bar{C}\left(v_{i}\right)=\{i-2, i-1\} i=2,3, \cdots n$;

Obviously, the $f$ is $(n+3)-A V D E C$ of $M\left(S_{n}\right)$, the conclusion is true.

From all of above, the conclusion is true.

From the conclusions of Theorem 1,2,3,4,5,6, the Conjecture is true for equal degree graph.

\section{References}

1. Harary F, Conditional colorability in graphs, In Graphs and Applications,Proc Graph theory Fist Colorado symp,John Wilh \& Sons, New York, 1985;

2. J.cerny M.hornak and R.sotak Observability of a graph, Math. Slovaca 46, 1996,21-31;

3. Burris A C and Schelp R H, Vertex-distinguishing Proper Edge-colorings, J of Graph Theory, 1997, 26(2):73-82;

4. Bazgan C Harkat-Benhamdine A, Li H, etc. On the Vertex-distinguishing Proper Edge-coloring of Graph, J Combin Theory Ser B, 1999, 75:288-301;

5. Balister P N, Bollob'as B, Schelp R H, Vertex distinguishing coloring of graphs with $\Delta(\mathrm{G})=2$, Discrete Mathematics,2002,252(2):17 29;

6. Balister $\mathrm{P}$ N,Riordan $\mathrm{O} M$ and Schelp $\mathrm{R} \mathrm{H}$, Vertex-distinguishing edge coloring of graphs, J. Graph Theory 42(2003)95-109;

7. Zhongfu Zhang, Linzhong Liu, Jianfang Wang, Adjacent Strong Edge Coloring of Graphs, Applied MathematicsLetters, 2002, 15:623-626;

8. Wang shudong, $\mathrm{Li}$ chongming, $\mathrm{Xu} \mathrm{Ji}$, et, On the adjacent strong edge coloring of some graphs, $\mathrm{J}$ of Mathematical Research and exposition, Vo1.22, No 4, 2002, 412-417;

9. Balister $\mathrm{P} N$, Gyori and Schelp R H, On the adjacent-strong edge coloring of graphs with $\Delta(\mathrm{G}) \leq 3$, J.G.T to appear;

10. Bondy J A and Marty U S R, Graph Theory with Applications, The Macmillan Press Ltd, New York, 1976;

11. Chartrand G, Linda L F, Graphs and diagraphs, Ind edition wadsirth Brokks/cole, Monterey, CA(1986). 